Revista Ingeniería Biomédica

ISSN 1909-9762 / Volumen 9 / Número 18 / Julio-diciembre de 2015 / pp. 81-87

Escuela de Ingeniería de Antioquia-Universidad CES / Envigado, Colombia

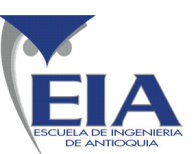

Ser. Saber y Serwir

\title{
Manual para la Gestión del Mantenimiento Correctivo de Equipos Biomédicos en la Fundación Valle del Lili
}

\author{
D.F. Primero ${ }^{1,2}$, J.C. Diaz ${ }^{1}$, L.F. García², A. González-Vargas ${ }^{1, \psi}$ \\ ${ }^{1}$ Grupo GIEIAM, Facultad de Ingeniería, Universidad Santiago de Cali, Colombia \\ ${ }^{2}$ Fundación Valle del Lili, Cali, Colombia
}

Recibido 1 de julio de 2015. Aceptado 15 de agosto de 2015

\section{Manual for the Management of Corrective Maintenance of Biomedical EQUIPMENT IN Fundación VALLE dEL LiLI}

\author{
Manual de Gestão da MANUTENÇ̃̃o CORRETIVA DE EQUiPAMENTOS BIOMÉDiCOS
}

NA FundaČ̃o Valle del LiLi

\begin{abstract}
Resumen - El mantenimiento correctivo está orientado a corregir los defectos observados en los equipamientos o instalaciones, localizando las averías y reparándolas. Los costos de reparación de un equipo suelen ser mucho mayores en la etapa correctiva que en la etapa preventiva, y la literatura existente está orientada más hacia la prevención que hacia la corrección. Sin embargo, es inevitable que en una institución hospitalaria, como en cualquier otra, se presenten frecuentemente problemas en los equipos que no pudieron ser previstos o evitados mediante el mantenimiento preventivo o correctivo. Por esta razón, se hace necesario contar con protocolos para el mantenimiento correctivo que contengan criterios y procesos claramente definidos para mejorar la eficiencia y la eficacia en la solución de los problemas y que permitan la máxima disponibilidad en la prestación de los servicios. En este trabajo se presenta, como caso de estudio, el desarrollo e implementación de un Manual para la Gestión del Mantenimiento Correctivo en una de las clínicas de más alto nivel en la ciudad de Cali: La Fundación Valle del Lili.
\end{abstract}

Palabras clave - Mantenimiento correctivo; equipos biomédicos; gestión de mantenimiento.

Abstract - The corrective maintenance is aimed at correcting the shortcomings in the equipment or facilities, locating faults and repairing them. Repair costs of equipment are usually much higher in the corrective stage than in the preventive stage, and the existing literature is oriented more towards prevention than correction. However, it is inevitable that in a hospital, as elsewhere, problems are often present on computers that could not be foreseen or avoided through preventive or corrective maintenance. For this reason corrective maintenance protocols containing clearly defined criteria and processes to improve efficiency and effectiveness in solving problems and ensuring maximum availability in the provision of services, are needed. This paper presents a case study, development and implementation of a Management Manual for corrective maintenance of the highest level clinics in the city of Cali: The Fundación Valle del Lili.

\% Dirección para correspondencia:agonzalez@usc.edu.co

DOI: http:/dx.doi.org/10.14508/rbme.2015.9.18.81-87 
Keywords - Corrective Maintenance; Biomedical Equipment; Maintenance Management.

Resumo - A Manutenção corretiva visa corrigir as deficiências do equipamento ou instalações, localizando falhas e reparálas. Os Custos de reparação de equipamento são geralmente muito maior na fase corretiva que na fase preventiva, e a literatura existente é mais orientada para a prevenção que para a correção. No entanto, é inevitável que em um hospital, como em outros lugares, muitas vezes se apresentam problemas nos equipamentos que não podiam ser previstos ou evitados através da manutenção preventiva ou corretiva. Por esta razão, é necessário ter protocolos de manutenção corretiva que contenham critérios e processos claramente definidos para melhorar a eficiência e eficácia na resolução de problemas e para assegurar a máxima disponibilidade na prestação de serviços. Este trabalho se apresenta como um caso de estudo, o desenvolvimento e a implementação de um Manual de Gestão para manutenção corretiva das clínicas de maior nível na cidade de Cali: a Fundação Valle del Lili.

Palavras-chave - Manutenção corretiva; Equipamentos biomédicos; Gestão de manutenção.

\section{INTRODUCCIÓN}

$\mathrm{L}_{\mathrm{s}}^{\mathrm{a}}$ a gestión de mantenimiento es un elemento de gran apoyo en la dirección, control y manutención de la tecnología biomédica en el entorno hospitalario. Esta nos permite aumentar la calidad de los servicios prestados y maximizar la relación costo-beneficio en el funcionamiento del hospital [1].

La estandarización de los procesos para el seguimiento y control en los equipos biomédicos, al igual que una correcta ejecución de los procedimientos de mantenimiento correctivo, son una herramienta fundamental de la gestión de mantenimiento en el hospital y ayudan a minimizar los tiempos de reparación y vuelta al servicio de los equipos, maximizando con esto su disponibilidad.

En la gestión de mantenimiento, se distinguen dos tipos de actividades primordiales: actividades de mantenimiento preventivo y actividades de mantenimiento correctivo. El Mantenimiento Preventivo (MP) se compone de una serie de inspecciones periódicas de los equipos, haciendo tareas de limpieza, lubricación, ajuste, comprobación y reemplazo de componentes defectuosos, con el fin de mantener el equipo funcionando siempre en un estado óptimo. El Mantenimiento Correctivo (MC) es el trabajo realizado sobre un equipo para restaurar su estado operacional luego de presentar una falla. Este tipo de mantenimiento no es planificado, y solo se lleva a cabo a partir del reporte que hace el usuario del equipo o el personal que realiza el mantenimiento programado.

El mantenimiento preventivo ha sido descrito extensamente en diferentes textos (por ejemplo, [1-4]), pero la información acerca del mantenimiento correctivo es considerablemente menor y menos estandarizada. En una encuesta reciente, realizada por la Universidad Santiago de Cali (USC) en once Instituciones Prestadoras de Servicios de Salud (IPS) de mediana y alta complejidad en la ciudad de Cali y alrededores [5], se encontró que más de la mitad de las instituciones no contaba con un manual que definiera claramente los procesos e interacciones necesarias para la adecuada gestión del mantenimiento correctivo. La muestra dos resultados importantes al respecto: el primero es que sólo cuatro de las IPS reportan tener un protocolo o manual de mantenimiento correctivo, y el segundo es que sólo dos de las instituciones encuestadas reportan tener un índice de Prioridad para el Mantenimiento Correctivo $\left(\mathrm{P}_{\mathrm{MC}}\right)$, mientras que siete de ellas reportan tener dicho índice para el Mantenimiento Preventivo $\left(\mathrm{P}_{\mathrm{MP}}\right)$. De la misma manera que el $\mathrm{P}_{\mathrm{MP}}$ ayuda a clasificar los equipos médicos para determinar su inclusión o exclusión del inventario de mantenimiento [6], el $\mathrm{P}_{\mathrm{MC}}$ nos ayuda a definir el orden en que se realizarán las actividades de mantenimiento correctivo en caso de presentarse fallas simultáneas de varios equipos.

El mantenimiento correctivo es, en la práctica, significativamente más costoso que el mantenimiento preventivo [4], debido a las limitaciones de tiempo que obligan a tomar decisiones con poca planeación [7]. Sin embargo, es innegable que, sin importar lo bien que se planeen las actividades de mantenimiento preventivo, revisión y calibración, siempre existirán casos en que la falla solo es detectada cuando las funciones principales del equipo se han visto comprometidas. En este caso, se hace necesario tener procedimientos de acción claramente definidos, con el fin de minimizar el impacto causado por la falla y el tiempo de paro del servicio.

Con el fin de contribuir a la práctica de la gestión de mantenimiento, mediante la definición de procesos que ayuden a mejorar la eficiencia y la eficacia de las actividades de mantenimiento correctivo, se presenta, como caso de estudio, este Manual para la Gestión del Mantenimiento Correctivo, el cual ha sido desarrollado colaborativamente entre la USC y la sección de mantenimiento de Equipos Biomédicos de la Fundación Valle del Lili (FVL). La FVL cuenta con una amplia gama de tecnología y equipamiento biomédico debido a su alto nivel de complejidad y a los procedimientos asistenciales que se realizan en la institución, y en la actualidad tiene un total aproximado de 4300 equipos biomédicos, repartidos en las diferentes áreas asistenciales de la institución. Debido al gran volumen de equipos, es una tarea difícil y dispendiosa garantizar la disponibilidad de la tecnología las 24 horas. Por esta razón, los autores esperan que este manual sirva como referencia para otras instituciones que decidan 
estandarizar y protocolizar sus labores de mantenimiento correctivo.
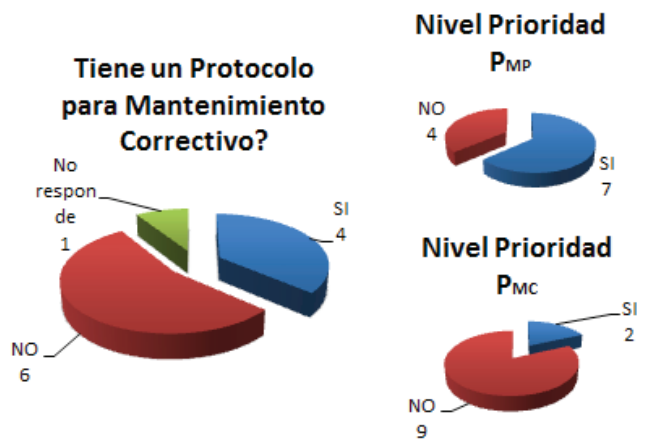

Fig. 1. Izquierda: Número de IPS que utilizan un protocolo o manual para el mantenimiento correctivo. Derecha (superior): Número de IPS que utilizan un nivel de prioridad para el mantenimiento preventivo $\mathrm{y}$ (inferior) correctivo.

\section{MetodologíA}

En el diagrama de la Fig. 2 se describen los procesos y actividades asociadas a la gestión del mantenimiento correctivo en la FVL. Los recuadros en línea verde indican quién es la persona o departamento encargado de realizar cada labor. Los recuadros en línea roja indican procesos que serán descritos con mayor detalle en las próximas secciones.

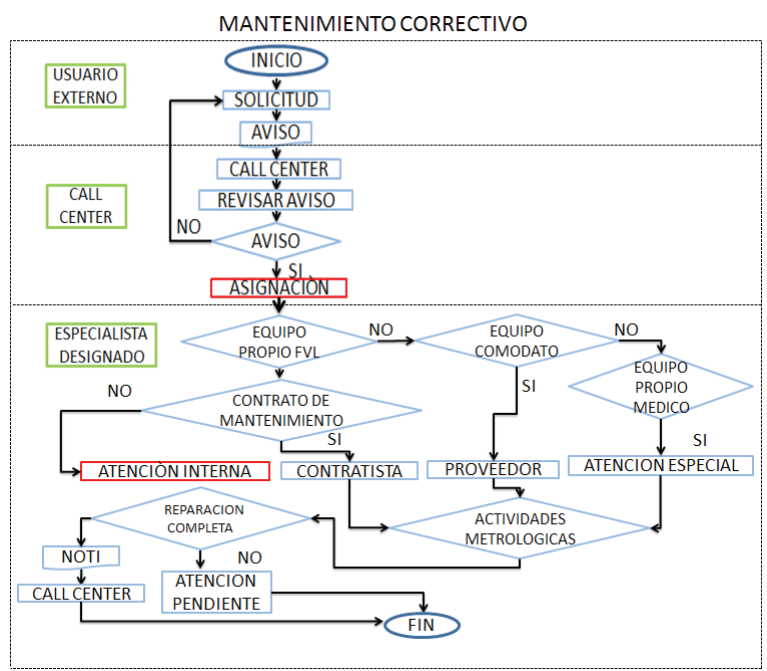

Fig. 2. Diagrama de flujo para la gestión del mantenimiento correctivo

\section{A. Solicitud}

El primer paso es la solicitud, la cual es realizada por el usuario ya sea mediante un aviso en SAP [8], o mediante una llamada al Call Center de mantenimiento. En cualquier caso debe indicarse la falla del equipo, su código de inventario, ubicación y nombre el usuario reportante.

\section{B. Asignación}

El segundo paso es la asignación, la cual está a cargo del Call Center de mantenimiento. Tal como se había mencionado en la sección I, establecer un índice de prioridad $\mathrm{P}_{\mathrm{MC}}$ ayuda a definir el orden en que se atenderán las solicitudes hechas al Call Center. El $\mathrm{P}_{\mathrm{MC}}$ del procedimiento es establecido con base en dos consideraciones: la categoría de urgencia del equipo y el orden de llegada de los avisos. Los equipos con categoría de urgencia 1 (ECU1) son: angiógrafos de cateterismo, equipos de cirugía, medicina nuclear, imágenes diagnósticas, radioterapia. Los equipos con categoría de urgencia 2 (ECU2) son: ventiladores mecánicos, máquinas de anestesia, desfibriladores y equipos de función pulmonar. La categoría del equipo puede variar de acuerdo a las condiciones del incidente, como por ejemplo el hecho de que la avería se presente durante un procedimiento o la existencia de un equipo de respaldo. Para la asignación de acuerdo al orden de solicitud se utiliza un proceso FIFO (first In-first Out). Este último criterio es el único que aplica para todos los reportes de avería de equipos que no entren en la clasificación descrita por la categoría de urgencia. El proceso de asignación de solicitudes por parte del Call Center se muestra en la Fig. 3. Cuando el Call Center haya definido el especialista encargado de atender la solicitud reportada, deberá informarle al mismo inmediatamente de la tarea asignada y la prioridad de la misma. Igualmente deberá generar el correspondiente reporte en SAP.

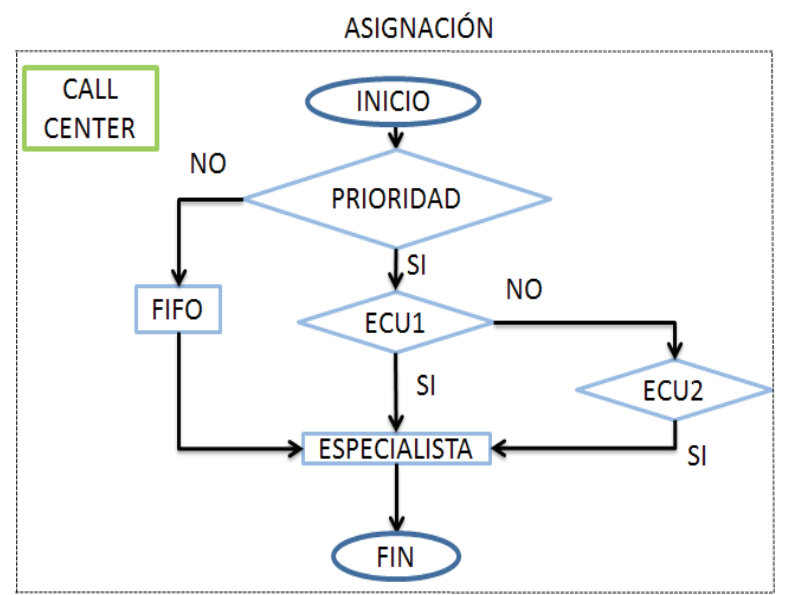

Fig. 3. Diagrama de flujo de la asignación de solicitudes de servicio de MC a cargo del Call Center de Mantenimiento.

\section{Atención}

Este proceso está a cargo del especialista de equipos biomédicos designado por el Call Center. Inicialmente, el encargado debe definir el proceso de atención apropiado de acuerdo al grupo al que pertenece el equipo reportado. 


\section{ATENCION INTERNA}

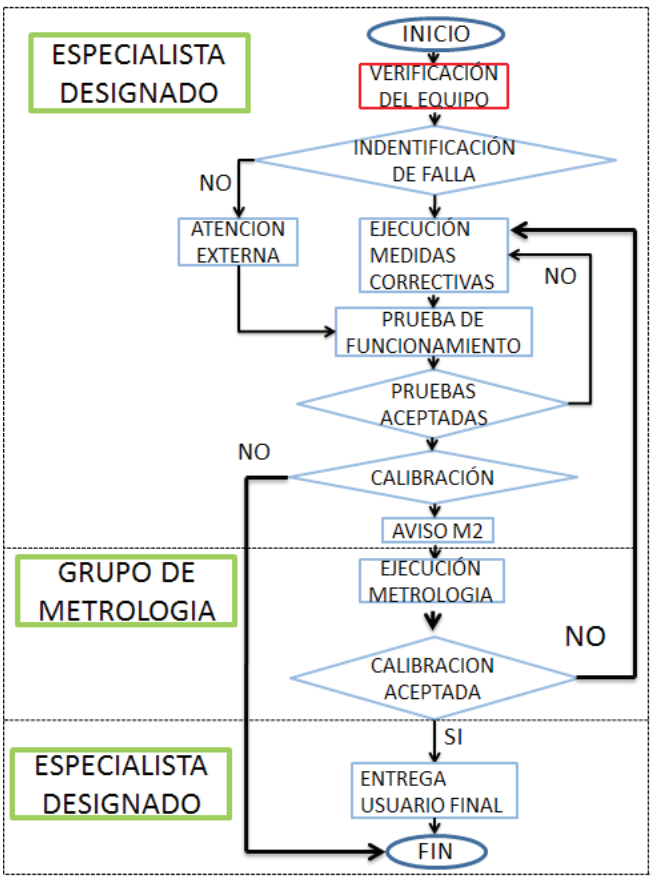

Fig. 4. Diagrama de flujo del proceso de Atención Interna.

El primer grupo son los equipos propios de la FVL, en cuyo caso se debe establecer si el equipo está en contrato de mantenimiento. Si es así, se debe contactar al contratista a cargo para que la solicitud sea atendida con brevedad en función del contrato establecido. En este caso, el equipo no debe ser intervenido por el personal de la sección de equipos biomédicos a menos que reciba la orientación y autorización del proveedor y solo siempre y cuando no requieran intervenciones mayores sobre el mismo. Cuando el equipo no se encuentra sujeto a un contrato de mantenimiento, la atención será realizada por el personal de mantenimiento correctivo del departamento (Fig. 4).

El primer paso de la atención interna es la verificación inicial (Fig. 5), con este procedimiento se determina si los daños son menores y no van más allá de ajustes físicos externos o modificaciones en la configuración del equipo, o si se trata de una avería mayor y se requieren procedimientos más complejos. Si el problema se resuelve a partir de la verificación inicial, el MC termina, y el equipo es entregado al usuario final.

Cuando la avería va más allá de la verificación inicial, el MC continúa, y se procede a tomar medidas correctivas basadas en los procedimientos de diagnóstico y reparación definidos por el manual de servicio del equipo, así como en la experiencia del especialista encargado de la actividad.

Después de la ejecución de las medidas correctivas, es necesario comprobar si el equipo queda funcionando de acuerdo a las especificaciones del fabricante. Esta comprobación es realizada por el grupo de Metrología, mediante procedimientos de calibración. Si el equipo pasa la actividad de calibración, se hace entrega del equipo al usuario y se procede a crear una notificación final de actividades (NOTI) e informar al Call Center del fin de las medidas correctivas.

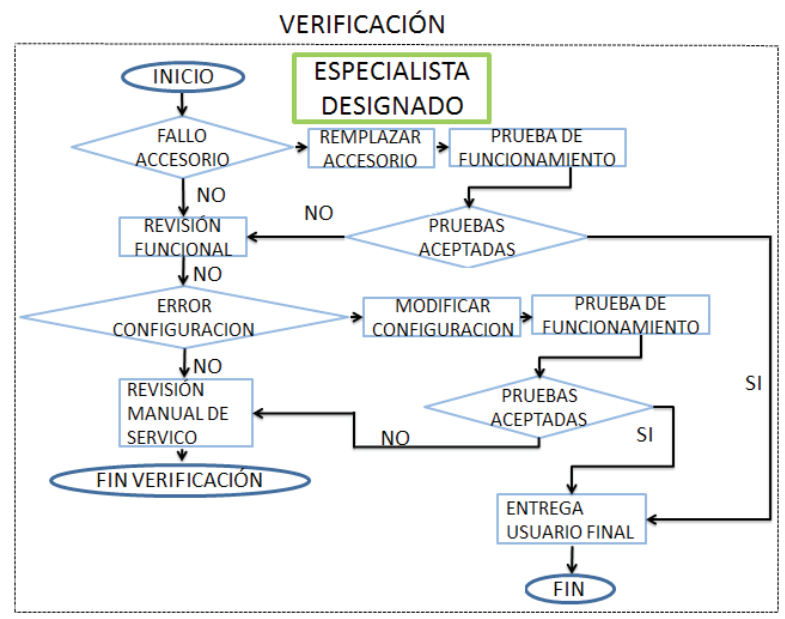

Fig. 5. Diagrama de flujo del proceso de Verificación del Equipo

En los casos en que las medidas tomadas en la atención interna no sean suficientes, se deberá considerar una atención externa (Fig. 6), contactando proveedores especializados en el equipo averiado. La tarea del proveedor consistirá en revisar el daño, emitir un diagnóstico y cotizar la reparación. El costo de reparación será evaluado por el Coordinador de Equipos Biomédicos y el especialista designado. Si el costo de reparación excede el 50\% del valor de reposición se deberá diligenciar un Reporte de Evento o Daño mayor mediante el cual se definirá si el equipo será reparado o será dado de baja.

Cuando se determina que el equipo será dado de baja, se debe entregar una copia del Reporte de Evento o Daño Mayor al usuario, para que este pueda iniciar los procesos de reposición respectiva (alquiler, compra, préstamo, etc). Adicionalmente se entrega otra copia del Reporte al líder del grupo de Capacitación e Ingreso de Tecnología para que se proceda con la baja del equipo en el sistema.

Cuando el daño no exceda el $50 \%$ del costo total del equipo, se tomarán las medidas para la ejecución de atención externa. La principal será verificar si la reparación es en sitio o no. Si la reparación es en sitio, entonces se agenda una cita para la visita del proveedor a la FVL. En el caso en que el proveedor requiere que el equipo sea enviado a sus instalaciones, el especialista designado deberá garantizar un apropiado proceso de empaque y transporte del equipo hasta las instalaciones del proveedor. Para proteger el equipo transportado de problemas durante 
este proceso, el especialista designado deberá asegurar el equipo, con la compañía definida por la FVL, por el monto más cercano posible al valor comercial del equipo. El especialista designado deberá además notificar al proveedor del envío del equipo y debe hacer seguimiento a la recepción del mismo por parte del proveedor cerciorándose que el equipo llegó a su destino.

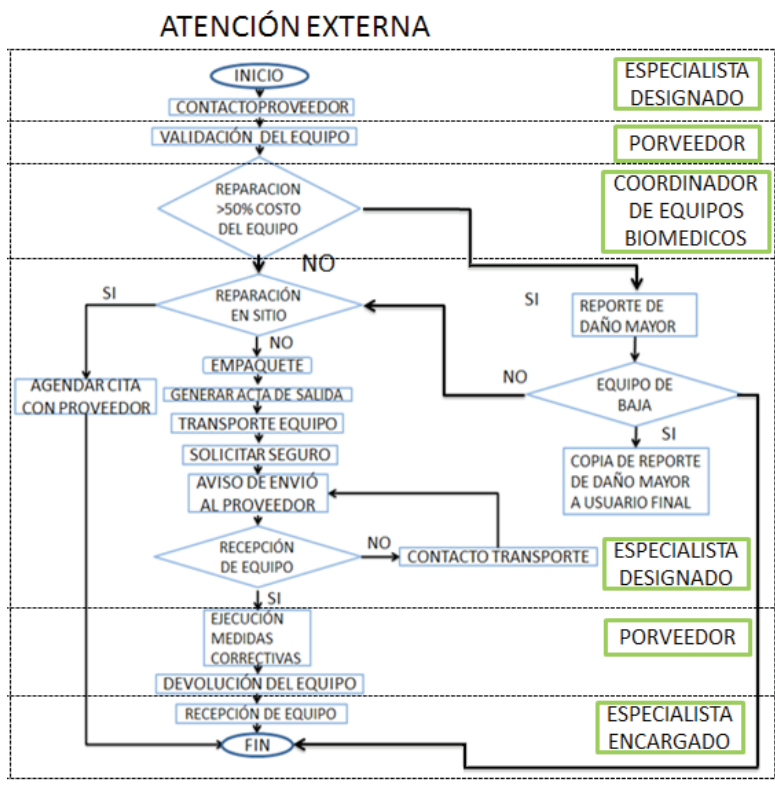

Fig. 6. Diagrama de flujo de la actividad de Atención Externa.

Como se especifica en el diagrama de flujo de mantenimiento correctivo (Fig. 2), cuando el equipo no es propiedad de la FVL se realiza otro tipo de atención. Si el equipo está en Comodato, el especialista designado debe contactar al proveedor propietario del equipo para que este se encargue de solucionar la avería del equipo. Si el equipo Pertenece a un Médico de FVL, se tendrá en cuenta el daño producido y el costo de la reparación, sé evaluarán las opciones de reparación para el equipo y si esta puede ser realizada por el especialista designado. Si la reparación excede el costo establecido por la FVL para los equipos propiedad de médicos, entonces será el propietario quien deberá buscar una solución externa. Si se logra la reparación completa de la avería, se cierra el MC y se genera una NOTI, dando aviso previamente al Call Center.

$\mathrm{Si}$ se produjeron inconvenientes que impidieron la finalización de la reparación, entonces se generará una notificación pendiente (NOTP) y se informará al usuario final el estado en el que queda el equipo, este proceso se denomina Atención Pendiente (Fig. 7). Una de las causas por las cuales el trabajo no se completó en su totalidad puede ser la falta de repuestos en el stock interno. Cuando este es el caso, se evalúa la adquisición de repuestos solicitando cotizaciones a las casas comerciales a través del departamento de Suministros, teniendo en cuenta que la decisión final de adquisición dependerá de la sección de Equipos Biomédicos en cabeza de su Coordinador, después de analizar cada una de las proformas obtenidas. Si el repuesto a adquirir es un repuesto nuevo, este debe ser codificado e incluido dentro de la base de datos existente en SAP para el departamento de suministros. Si no se obtiene una respuesta inmediata por parte del Departamento de Suministros, se hará seguimiento semanalmente por parte del especialista designado para que el proceso sea agilizado. El sub-proceso logístico de adquisición se muestra en la Fig 8.

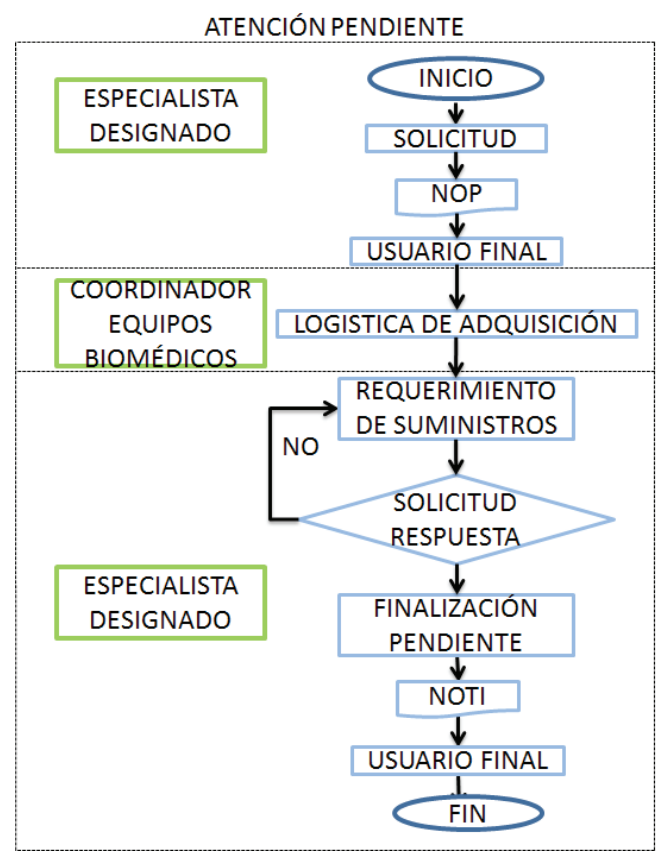

Fig. 7. Diagrama de flujo de la actividad de Atención Pendiente.

Cuando se cuenta con todos los medios necesarios para la conclusión de los medidas correctivas pendientes (repuesto o servicios), se ejecuta la actividad, se entrega el equipo a la sección de Metrología para que ejecute la calibración correspondiente y se entrega al usuario final. Posteriormente se notificará oficialmente al usuario final mediante un correo electrónico que las actividades faltantes fueron terminadas. Al momento de finalización de las actividades se debe generar una NOTI. El proceso de diligenciamiento de las NOTP y NOTI, involucran los mismos parámetros de información, únicamente diferenciándose en la indicación de notificación final. La notificación incluirá los siguientes campos:

- Especialista que desarrolló la actividad (Ingeniero, Tecnólogo o Proveedor)

- Número de personal del especialista designado (Si es interno). 
- $\quad$ Tiempo total de la activad realizada. Puede ser la suma de tiempos parciales.

- La fecha y hora de inicio de las actividades

- La fecha y hora de fin de las actividades

- Actividades realizadas

- $\quad$ Observaciones (Es el indicador que diferencia si es una NOTP o una NOTI)

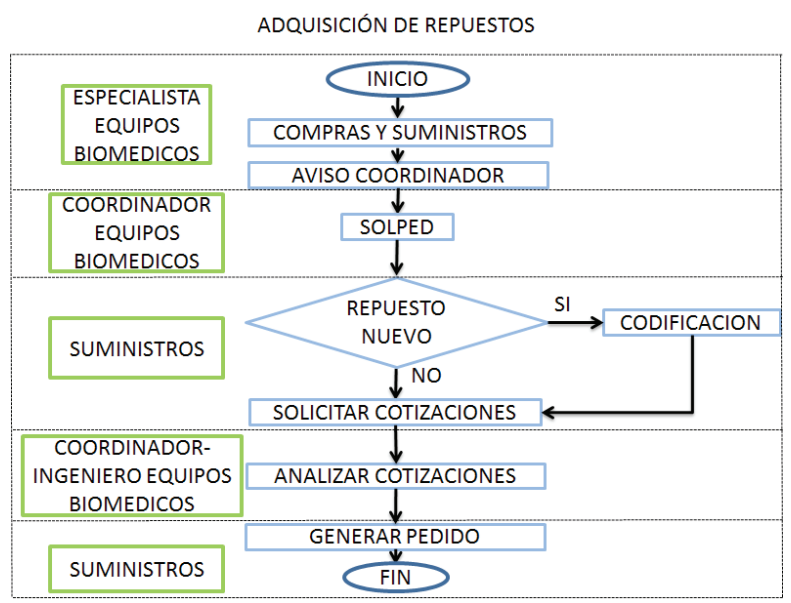

Fig. 8 Diagrama de flujo de la actividad de Adquisición de Repuestos.

\section{RESULTADOS}

La cuantificación de las mejoras producidas por este manual de mantenimiento correctivo sólo es posible a través de los indicadores de cumplimiento del servicio. Dichos indicadores han estado disponibles durante los últimos tres años gracias a las mejoras que ha hecho la sección de mantenimiento de Equipos Biomédicos. La Fig. 9 muestra el número de solicitudes de mantenimiento correctivo anuales para los años 2012, 2013 y 2014, junto con los respectivos índices de cumplimiento. La implementación de los procedimientos del manual de mantenimiento correctivo sólo se ha realizado a partir del año 2014; sin embargo, es notable que el índice de cumplimiento en dicho año fue del $100 \%$, mientras los años precedentes muestran índices inferiores aunque buenos.

\section{Discusión}

El Manual para la Gestión del Mantenimiento Correctivo de la Fundación Valle del Lili es un producto que se encuentra en constante desarrollo, y en el futuro cercano planea hacer mejoras importantes gracias a la introducción de nuevos factores que permitan volver cada vez más cuantificable la labor del departamento y el efecto de las averías en los equipos. Una de dichas mejoras a corto plazo es la inclusión de una ecuación que permita calcular el $\mathrm{P}_{\mathrm{MC}}$ de manera más exacta que la actual categorización. Igualmente, se planea obtener mejoras utilizando indicadores del tiempo de ejecución de las actividades correctivas, así como índices de disponibilidad de los equipos e indicadores de las causas de falla más comunes.

\section{Solicitudes de mantenimiento correctivo al año}

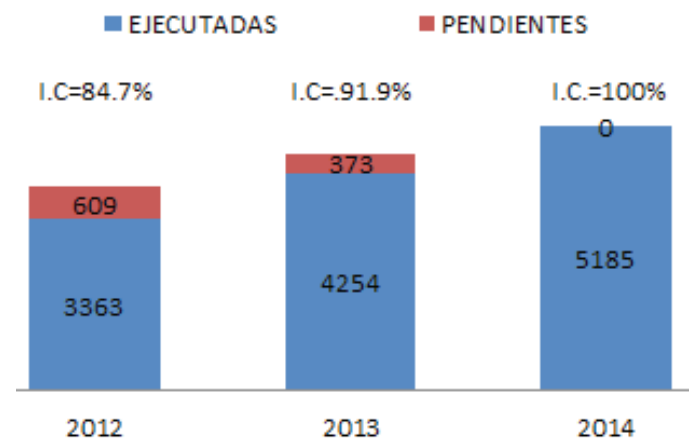

Fig. 9. Número de solicitudes de mantenimiento correctivo ejecutadas y pendientes en los años 2012-2014.

\section{CONCLUSIÓN}

Este manual provee un punto de partida para la protocolización y sistematización del mantenimiento correctivo en las instituciones hospitalarias. Si bien se ha demostrado que la gestión de mantenimiento brinda mejores resultados cuando se orienta hacia la prevención y no hacia la reparación, también es claro que los fallos ocasionales de los equipos biomédicos son imposibles de erradicar debido a la multitud de factores que pueden generarlos. De acuerdo a esto, los autores presentan este manual como una estrategia efectiva para estar preparados contra dichos eventos, teniendo como objetivo final garantizar la seguridad del paciente y minimizar las consecuencias adversas en la prestación de los servicios del hospital.

\section{AgradeCIMIENTO}

Los autores expresan su reconocimiento y agradecimiento a la Fundación Valle del Lili, en especial al departamento de mantenimiento de equipos biomédicos, por su colaboración y ayuda durante la realización de este trabajo.

\section{REFERENCIAS}

[1]. Rodríguez E., Ingeniería Clínica. (2003). En: http://bioingenieria. edu.ar/academica/catedras/ingenieria_clinica/archivos/TP2013/ ic-denis.pdf

[2]. Gross J. M. Fundamentals of Preventive Maintenance, Amacom, 2002.

[3]. Mobley K. An Introduction to Predictive Maintenance. Butterworth-Heinemann, 2002.

[4]. Mobley K. Maintenance Fundamentals. Butterworth-Heinemann, 2004. 
[5]. González-Vargas A., Collazos M., García L., Ladino J. Cano A., González S. Estado actual de la Ingeniería Clínica en las instituciones hospitalarias de Cali. Enviado para revisión al II Congreso Internacional de Ingeniería Clínica, Medellín, 2015.

[6]. Rodríguez E., Miguel A., Sánchez M. Gestión de Mantenimiento para Equipos Médicos. Memorias del II Congreso Latinoamericano de Ingeniería Biomédica, La Habana, 2001.

[7]. Higgins L., Mobley K. Maintenance Engineering Handbook, McGraw-Hill, 2008.

[8]. Hernández J., Martínez F., Keogh J. SAP R/3 Handbook, McGraw-Hill, 2005. 